\title{
Integrated Planning and Management of Survivable Wireless Communications Networks
}

\author{
Frank Yeong-Sung Lin and Chih-Yuan Lin \\ Department of Information Management \\ National Taiwan University \\ Taipei, Taiwan \\ Tel: $+886-2-2363-8423 \quad+886-2-2215-0350$
}

Fax: +886-2-2758-4773

Email: yslin@im.ntu.edu.twr6725007@im.ntu.edu.tw

Invited Paper

\begin{abstract}
In this paper, the problem of integrated planning and management of survivable wireless communications networks is investigated. A number of major issues in the planning and management process for wireless communications networks are hereinafter jointly considered. They are (i) Mobile telephone switching office (MTSO) allocation, (ii) MTSO interconnection and traffic routing (iii) base station allocation, (iv) base station transmission power control, (v) channel assignment and (vi) mobile station homing. Survivability issues are also considered such that the network is designed to survive pre-specified failure scenarios. We formulate the problem as a combinatorial optimization problem where the system installation cost and the channel licensing cost are minimized. The basic approach to the algorithm development is Lagrangean relaxation. In computational experiments, the proposed algorithm is shown to be effective and achieves up to $26 \%$ improvement in the total deployment cost over a primal heuristic.
\end{abstract}

\section{Introduction}

Wireless communications has received increasing attention recently due to the advances of technology and the evergrowing user demand. For system planners, managers and administrators, to plan and manage such complex systems in an efficient and effective way is always a challenge. Although intensive research on different areas of wireless communications network planning and management has been conducted, e.g., base station allocation, base station power control, and channel assignment, relatively little work attempting to attack the overall planning and management problem in an integrated and joint manner has been seen in the literature. Hao et. al. [1] considered the design and radio network resource planning problem for cellular mobile communications systems. The problem was to determine the cell number, cell site allocation, and the specific base station parameters in order to minimize the total system cost and to comply with the required system performances. They used a hierarchical optimization planning method (HOP) to deal with the problem and developed a three-level optimization approach. The problem was formulated as a large combinatorial optimization model and the simulated annealing approach was applied. Wu and Lin [2] first developed a mathematical formulation of and solution procedure to the aforementioned overall planning and management problem. However, survivability issues, MTSO interconnection and traffic routing were not considered in their work. Their algorithms based on Lagrangean relaxation were shown to be practically efficient and effective compared with benchmark heuristics.

This paper investigates the problem of wireless communications networks planning and management under QoS/GoS and survivability constraints. The problem is to decide suitable positions for placing communication devices, capacity assignment, traffic routing, channel assignment, and base station power control, etc. Due to the high and complicated correlation among these decisions, the overall optimality would be significantly compromised if the decisions are made independently or sequentially. It is then the main focus of this paper to formulate and to solve the problem in a joint fashion so that near global optimality can be achieved.

In order to better describe the problem, we highlight the following essential subproblems from the joint planning and management problem: (i) MTSO Allocation Subproblem: to decide where and in what capacity we should allocate the MTSO's to successfully handle the traffic introduced by their slave base stations. (ii) Base Station Allocation Subproblem: to concern about the allocation of the base stations in the designated service area. (iii) Power Control Subproblem: to decide the transmission power radiated from each base station to cover all the mobile stations. (iv) Network Topology and Capacity Assignment Subproblem: to determine the location and capacity of links between base stations and MTSO's, and links among MTSO's. (v) Homing and Routing Subproblem: to select a path for each mobile station O-D pairs. (vi) Channel Assignment Subproblem: to assign channels to each base station selected in the base station allocation subproblem.

At the network planning stage, designers often plan their networks based on the assumption that all the components are operational, and then treat error handling and recovering as another problem. However, network failures are indeed inevitable, and the system performance will degrades significantly upon the occurrence of such failures. We therefore consider survivability issues in planning and management of wireless communications networks. Any pre-specified failure scenario can be considered in the proposed model such that sufficient spare resources are allocated at system generation to recover from such possible failures. The system will regain feasibility upon each of these failure scenarios through base station transmission power readjustment, channel reassignment and traffic rerouting.

We model the joint wireless communications network planning and management problem considering survivability issues as a combinatorial optimization problem. To the best of our knowledge, the proposed approach is the first attempt to the 
said problem. Lagrangean relaxation and the subgradient method are applied to solve the problem.

The remainder of this paper is organized as follows. In Section II, a mathematical formulation of the problem is presented. In Section III, a dual approach to attacking the problem based on Lagrangean relaxation is proposed. We then propose a number of algorithms to calculate primal feasible solutions in Section IV. Computational results are finally reported in Section V.

\section{Problem Formulation}

The considered problem is modeled as a mixed integer programming problem. The objective function is to minimize the sum of the following items: (i) the cost of links, including connections among MTSO's and their slave base stations, and the cost related to MTSO's (MTSO is modeled as a link in our formulation), (ii) fixed and variable cost of base stations, including installation, operation, maintenance and equipment cost, and (iii) channel licensing cost. These items are the major costs involved in configuring a cellular network [3]

For the convenience of the reader, the notation used in the problem modeling is listed in the table below, followed by a mathematical formulation referred to as Problem (IP).

\begin{tabular}{|c|c|}
\hline Notation & Descriptions \\
\hline$c_{l}$ & Capacity assigned to link I (decision variable) \\
\hline$g_{j}^{e}$ & $\begin{array}{l}\text { Aggregate flow on base station } j \text { at network state } \\
e \text { (secondary decision variable) }\end{array}$ \\
\hline$h_{i}^{e}$ & $\begin{array}{l}\text { Decision variable which is } 1 \text { if channel } i \text { is used } \\
\text { at network state } e \text { and } 0 \text { otherwise }\end{array}$ \\
\hline$m$ & $\begin{array}{l}\text { Total number of channels required (decision } \\
\text { variable) for the network }\end{array}$ \\
\hline$n_{j}$ & Number of channels required for base station $j$ \\
\hline$x_{p}^{e}$ & $\begin{array}{l}\text { Routing decision variable which is } 1 \text { if path } p \text { is } \\
\text { selected at network state } e\end{array}$ \\
\hline$y_{j i}^{e}$ & $\begin{array}{l}\text { Decision variable which is } 1 \text { if channel } i \text { is } \\
\text { assigned to base station } j \text { at network state } e\end{array}$ \\
\hline$\alpha_{l}^{e}$ & $\begin{array}{l}\text { Call blocking probability requirement associated } \\
\text { with link } l\end{array}$ \\
\hline$\beta_{j}^{e}$ & $\begin{array}{l}\text { Call blocking probability requirement associated } \\
\text { with base station } j\end{array}$ \\
\hline$\delta_{p l}$ & $\begin{array}{l}\text { Indicator function which is } 1 \text { if link } l \text { is on path } p \\
\text { and } 0 \text { otherwise }\end{array}$ \\
\hline$\sigma_{l j}$ & $\begin{array}{l}\text { Indicator function which is } 1 \text { if base station } j \text { is } \\
\text { one end of link } l \text { and } 0 \text { otherwise }\end{array}$ \\
\hline$\lambda_{l r}$ & $\begin{array}{l}\text { Indicator function which is } 1 \text { if mobile station } r \\
\text { is one end of link } l\end{array}$ \\
\hline$Q_{e}$ & Coverage ratio requirement at network state $e$ \\
\hline$K_{w}$ & User demand of O-D pair $w$ (in Erlangs) \\
\hline$U$ & Unit channel licensing cost \\
\hline$D_{i j^{\prime}}$ & Distance between base station $j$ and $j$ \\
\hline$\overline{D_{r j}^{\prime}}$ & $\begin{array}{l}\text { Distance between mobile station } r \text { and base } \\
\text { station } j\end{array}$ \\
\hline$A_{l}\left(c_{l}\right)$ & Cost function of link $l$ with capacity $c_{l}$ \\
\hline$S_{j}\left(n_{j}\right)$ & Cost function of base station $j$ with capacity $n_{j}$ \\
\hline
\end{tabular}

(1) $\sum_{p \in P_{*}^{P}} x_{p}^{e}=0$ or 1

$\forall w \in W, e \in E$

(2) $0 \leq r_{j}^{e} \leq R_{j}$

$\forall j \in B, e \in E$

(3) $D_{r j}^{\prime} \sum_{w \in H} \sum_{p \in P_{i}^{i} j \in l_{3}} \lambda_{l r} \sigma_{l j} \delta_{p l} x_{p}^{e} \leq r_{j}^{e}$

$\forall r \in O, j \in B, e \in E$

(4) $\sum_{w \in W_{p \in P_{\psi}^{*}}} \sum_{w} x_{p}^{e} \delta_{p l} \leq E\left(c_{l}, \alpha_{l}^{e}\right)$

$\forall l \in L, e \in E$

(5) $Q_{e} \leq \frac{\sum_{w \in W} \sum_{p \in P_{w}^{*}} K_{w} x_{p}^{e}}{\sum_{w \in W} K_{w}}$

$\forall e \in E$

(6) $T\left(g_{j}^{e}, \beta_{j}^{e}\right) \leq \sum_{i \in F} y_{j}^{e}$

$\forall j \in B, e \in E$

(7) $\sum_{w \in W} \sum_{p \in P_{v}^{j} \in L_{3}} K_{w} \sigma_{l j} \delta_{p l} x_{p}^{e} \leq g_{j}^{e}$

$\forall j \in B, e \in E$

(8) $y_{j i}^{e} \leq h_{i}^{e}$

$\forall i \in F, j \in B, e \in E$

(9) $\sum_{i \in F} y_{j i}^{e} \leq N$

$\forall j \in B, e \in E$

(10) $\sum_{i \in F} y_{j i}^{e} \leq n_{j}$

$\forall j \in B, e \in E$

(11) $\sum_{i \in F} h_{i}^{e} \leq m$

$\forall e \in E$

(12) $\sum_{\substack{j \in \in \\ j \neq i}}\left(\frac{r_{f}^{e}}{D_{j^{\prime}}}\right)^{t} y_{f^{e}}^{e} \leq G_{j}+\left(\frac{1}{\tau}-G_{j}\right) y_{j i}^{e} \quad \forall i \in F, j \in B, e \in E$ 


$$
\begin{array}{lr}
\text { (13) } h_{t}^{e}=0 \text { or } 1 & \forall i \in F, e \in E \\
\text { (14) } x_{p}^{e}=0 \text { or } 1 & \forall p \in P_{w}^{e}, w \in W, e \in E \\
\text { (15) } y_{j i}^{e}=0 \text { or } 1 & \forall i \in F, j \in B, e \in E \\
\text { (16) } c_{l} \in V_{0} & \forall l \in L_{0} \\
\text { (17) } c_{l} \in V_{1} & \forall l \in L_{1} \\
\text { (18) } c_{l} \in V_{2} & \forall l \in L_{2} \\
\text { (19) } n_{j} \leq N & \forall j \in B \\
\text { (20) } n_{j} \in Z^{+} & \forall j \in B \\
\text { (21) } m \leq M & \\
\text { (22) } m \in Z^{+} . &
\end{array}
$$

Physical meanings of the constraints are briefly described as follows. Constraint (1) (with Constraint (14)) requires that the traffic for an O-D pair either be transmitted over exactly one path, or not be transmitted (rejected by the system). Constraint (2) is to ensure that the transmission radius of each base station $j$ be between 0 and $R_{j}$. Constraint (3) requires that a mobile station be in the service area of a base station before being served by that base station. Constraint (4) requires that the number of channels allocated to link $l$ be sufficient to result in a call blocking probability not exceeding a pre-specified threshold. Constraint (5) requires that the service coverage upon every pre-specified failure scenario satisfy the required threshold. Constraints (6) and (7) are to ensure that the number of channels assigned to each base station be large enough to serve the traffic demand of its slave mobile stations. Constraint (8) is to ensure that a channel should be activated (licensed) before it can be assigned to a base station. Constraint (9) requires that the number of channels assigned to each base station at every system state (failure scenario) be no greater than a pre-specified bound. Constraint (10) requires that $n_{j}$ be the largest number of channels required for base station $j$ among all system states. Constraint (11) requires that $m$ be the largest number of channels required among all system states. Constraint (12) requires that for each channel, the aggregate cochannel interference be no greater than the pre-specified threshold.

Constraints (13), (14) and (15) require that the integer property of the decision variables with respect to channel usage, routing, and channel assignment, respectively, be satisfied. Constraints (16) to (18) require that the capacity of three types of links be chosen from the three given sets, respectively. Constraints (19) and (20) require that decision variable $n_{j}$ be a nonnegative integer and no greater than $N$. And Constraints (21) and (22) require that decision variable $m$ be a nonnegative integer and no greater than $M$.

\section{Solution Approach}

Lagrangean relaxation is a mathematical technique originally designed for solving large-scale linear programming problems in the 1970s [4,5]. It has later been applied to a large number of well-known integer programming problems and shown efficient and effective (e.g. the traveling salesman problem). The procedures of applying the Lagrangean relaxation method are as follows: Relax complicating constraints, multiple the relaxed constraints by corresponding Lagrangean multipliers, and add them to the primal objective function. By using the Lagrangean relaxation method, we can transform the primal problem (IP) described in Section 3.3 into the following Lagrangean relaxation problem (LR) where Constraints (3)-(8), (10) and (12) are relaxed.

\section{Problem (LR)}

$$
Z_{D}\left(a_{r j e}^{1}, a_{l e}^{2}, a_{e}^{3}, a_{j e}^{4}, a_{j e}^{5}, a_{j e}^{6}, a_{j e}^{7}, a_{j e}^{8}\right)=
$$

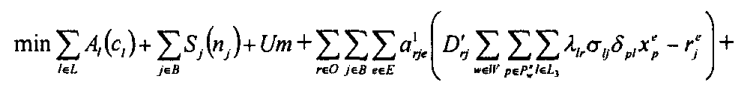

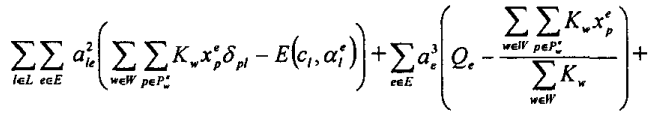$$
\sum_{j \in B} \sum_{\in \in E} a_{j e}^{4}\left(T\left(g_{j}^{e}, \beta_{j}^{e}\right)-\sum_{i \in F} y_{j i}^{e}\right)+\sum_{j \in B} \sum_{e \in E} a_{j e}^{j}\left(\sum_{w \in W} \sum_{p \in P_{\nu} / \epsilon L_{i}} K_{\Downarrow} \sigma_{l} \delta_{p l} x_{p}^{e}-g_{j}^{e}\right)+
$$$$
\sum_{i \in F} \sum_{j \in B} \sum_{e \in E} a_{i j e}^{6}\left(y_{j i}^{e}-h_{i}^{e}\right)+\sum_{j \in B} \sum_{e \in E} a_{j e}^{?}\left(\sum_{i \in F} y_{j i}^{e}-n_{j}\right)+
$$$$
\sum_{i \in F} \sum_{j \in B} \sum_{r \in E} a_{i j e}^{s}\left(\sum_{\substack{j \in B \\ j \neq j}}\left(\frac{r_{j}^{e}}{D_{i j^{\prime}}}\right)^{\prime} y_{j t}^{e}-G_{j}+\left(\frac{1}{\tau}-G_{j}\right) y_{j j}^{*}\right)
$$

s.t.: (1), (2); (9), (11), (13)-(22).

(LR) can be further decomposed into six independent subproblems. All of them can be optimally solved efficiently.' According to the weak Lagrangean duality theorem, for any $\left(a_{i j e}^{1}, a_{l e}^{2}, a_{e}^{3}, a_{j e}^{4}, a_{j e}^{5}, a_{i j e}^{6}, a_{j e}^{7}, a_{i j e}^{8}\right) \geq 0, \quad Z_{D}\left(a_{r e}^{1}, a_{l e}^{2}, a_{e}^{3}, a_{j e}^{4}, a_{j e}^{3}, a_{i e}^{6}, a_{j e}^{7}, a_{i e}^{8}\right)$ is a lower bound on $Z_{I P}$. [4]. We then apply the subgradient method [6] to calculate the tightest lower bound. Meanwhile, algorithms to be described in the next section are developed to calculate good primal feasible solutions.

\section{Getting Primal Feasible Solutions}

In addition to serving as a bounding procedure of large-scale optimization problems, the Lagrangean relaxation method usually provides solutions which, although may not be primal feasible, yet may shed light on the search process for good primal solutions $[1,7]$. In addition, the multipliers, which possess specific physical meanings, may also be applied to lead to good primal solutions. Both techniques are used in the development of algorithms for calculation primal feasible solutions in this paper.

Due to the complexity of the primal problem, a divide-andconquer strategy is proposed to calculate primal feasible solutions. As mentioned in Section 1, the overall joint problem can be considered as the combination of a number of subproblems, which can be further grouped into 4 main parts: (i)

\footnotetext{
$1,2,3$ Detailed algorithms are omitted due to the length limitation of the paper. A complete version of the paper is available upon request.
} 
base station allocation, power control, and mobile station homing subproblems, (ii) channel assignment subproblem, (iii) MTSO allocation subproblem and (iv) MTSO interconnection and traffic routing subproblems. Four algorithms ${ }^{2}$ are then proposed to solve these subproblems related to decision variables $x_{p}^{e}, n_{j}, y_{j i}^{e}$ and $r_{j}^{e}$, respectively. As mentioned previously, the algorithms are principally based upon implications of the Lagrangean results as guidelines to search optimal primal solutions. The proposed algorithms are tested in the computational experiments.

\section{Computational Experiments}

In this section, computational experiments are performed to evaluate the effectiveness of the proposed algorithms. For comparison purposes, a primal approach referred to as Algorithm 5.1 is developed. Solutions calculated by Algorithm 5.1 (without considering the pre-specified failure scenarios) and solutions by the proposed algorithm based upon Lagrangean relaxation are fed into the same procedure ${ }^{3}$ to calculate primal feasible solutions considering survivability constraints.

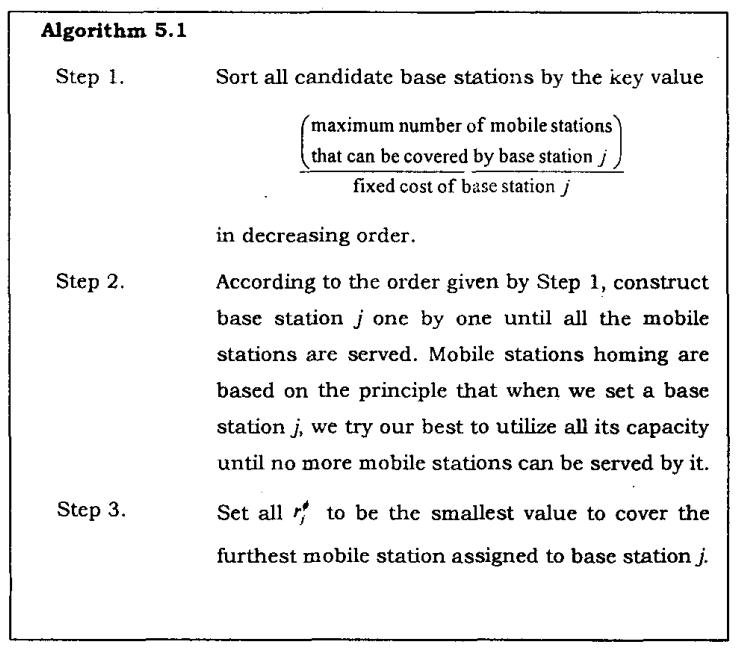

Two sets of experiments are conducted, where 10 and 18 cadidate base ststions are considered, respectively. The service area is assumed to be $100 \mathrm{~km}^{2}$ and the mobile station density is $0.0001 \mathrm{MS} / \mathrm{m}^{2}$. We also assume that call arrivals can be characterized by a Poisson process and the demand is randomly distributed in the service area. The demand generated by each mobile station is assumed to be 0.1 Erlang and the call blocking requirement for each base station is assumed to be $5 \%$. The fixed cost of a base station is randomly drawn from 4,000 to 6,000 thousand NT dollars. Details of other parameters are available upon request.

All the experiments are performed on a Pentium II $400 \mathrm{PC}$ running Microsof ${ }^{\circledR}$ Windows 98 with 64MB DRAM. The code is written in ANSI $\mathrm{C}$ and is compiled by Microsoft ${ }^{\mathbb{Q}}$ Visual C++ 6.0. Each iteration runs within seconds (averaged $14.38 \mathrm{sec}$. for 10 -node cases and $50.48 \mathrm{sec}$. for 18-node cases). Analysis of time complexity indicates that the growth of computation time is super-linear with respect to the number of candidate nodes.

The Lagrangean relaxation based algorithm and the primal based one are termed "BCM_L" and "BCM_P", respectively. For $\mathrm{BCM} \mathrm{L}$, the best result obtained in 1000 iterations is recorded and reported. Tables 5.1 and 5.2 summarize the computational results.

Tabie 5.1 Cost Comparison (10 Nodes)

\begin{tabular}{|c|c|c|c|c|c|c|c|c|c|c|}
\hline & 1 & 2 & 3 & 4 & 5 & 6 & 7 & 8 & 9 & 10 \\
\hline $\begin{array}{c}\text { Algarithm__ } \\
\text { BCM_L }\end{array}$ & 46624 & 47195 & 46532 & 41715 & 46624 & 52012 & 52012 & 41144 & 46624 & 46624 \\
\hline BCM_P & 52012 & 52012 & 46532 & 52012 & 52012 & 52012 & 52012 & 52012 & 52012 & 52012 \\
\hline $\begin{array}{c}\text { Improvement } \\
(\%)\end{array}$ & 11.56 & 10.21 & 0 & 24.68 & 11.56 & 0 & 0 & 26.41 & 11.56 & 11.56 \\
\hline
\end{tabular}

Table 5.2 Cost Comparison (18 Nodes)

\begin{tabular}{|c|c|c|c|c|c|c|c|c|c|c|}
\hline $\begin{array}{c}\text { CASE \# } \\
\text { Algorithm }\end{array}$ & 1 & 2 & 3 & 4 & 5 & 6 & 7 & 8 & 9 & 10 \\
\hline BCM_L & 51505 & 47395 & 51018 & 51018 & 51505 & 46606 & 51018 & 51018 & 45468 & 55917 \\
\hline BCM_P & 61958 & 55969 & 50581 & 61075 & 55275 & 55969 & 50696 & 61958 & 50696 & 55969 \\
\hline $\begin{array}{c}\text { Improvement } \\
(\%)\end{array}$ & 20.30 & 18.09 & -0.86 & 19.71 & 7.32 & 20.09 & -0.63 & 21.44 & 11.50 & 0.09 \\
\hline
\end{tabular}

From the computational results, it is observed that the Lagrangean relaxation based algorithms achieve up to $26.41 \%$ and $20.30 \%$ improvement over the primal based heuristics for two sets of experiments, respectively. This clearly demonstrates the effectiveness of the Lagrangean approach.

\section{References}

[1] Q. Hao et. al., "A Low-Cost Cellular Mobile Communication System: A Hierarchical Optimization Network Resource Planning Approach," IEEE Journal on Selected Areas in Communications, Vol. 15, No. 7, pp. 1315-1326, September 1997.

[2] S. Y. Wu and F. Y.-S. Lin, "Design and Management of Wireless Communications Networks," Proc. INFORMS CIST'99, pp. 284-306, Cincinnati, USA, May 1999.

[3] B. Gavish and S. Sridhar, "Economic Aspect of Configuring Cellular Networks," Wireless Networks, Vol. 1, No. 1, pp.115-128, February 1995.

[4] A. M. Geoffrion, "Lagrangean Relaxation and Its Uses in Integer Programming," Math. Programming Study, Vol.2, pp. 82-114, 1974.

[5] M. L. Fisher, "The Lagrangean Relaxation Method for Solving Integer Programming Problems." Management Science, Vol. 27, No. 1, pp. 1-18, January 1981.

[6] M. Held, P. Wolfe and H. D. Crowder, "Validation of Subgradient Optimization," Math. Programming, Vol. 6, pp. 62-88, 1974.

[7] F. Y.-S. Lin, "Quasi-static Channel Assignment Algorithms for Wireless Communications Networks," Proc. ICOIN'98, Japan, January 1998. 